La revue La revue pour l'histoire du CNRS

POUR LHISTORE DU CNRS $\quad 20 \mid 2008$

Aventures et recherches aux pôles

\title{
Alerte en Antarctique
}

où l'ozone stratosphérique fut menacé...

Marie-Lise Chanin

\section{CpenEdition}

Journals

Édition électronique

URL : https://journals.openedition.org/histoire-cnrs/5932

DOI : 10.4000/histoire-cnrs.5932

ISSN : 1955-2408

Éditeur

CNRS Éditions

Édition imprimée

Date de publication : 3 avril 2008

ISBN : 978-2-271-06562-9

ISSN : 1298-9800

Référence électronique

Marie-Lise Chanin, «Alerte en Antarctique », La revue pour l'histoire du CNRS [En ligne], 20 | 2008, mis en ligne le 03 avril 2010, consulté le 20 mai 2021. URL : http://journals.openedition.org/histoire-cnrs/ 5932 ; DOI : https://doi.org/10.4000/histoire-cnrs.5932

Ce document a été généré automatiquement le 20 mai 2021

Comité pour l'histoire du CNRS 


\title{
Alerte en Antarctique
}

\author{
Où l'ozone stratosphérique fut menacé...
}

Marie-Lise Chanin

Difficile de retracer l'histoire de la recherche dans les régions polaires sans évoquer la destruction de l'ozone stratosphérique et sans en expliquer les causes et les conséquences. Rappelons en préambule que l'ozone est un constituant présent en très faible quantité dans l'atmosphère, mais que sa présence a été essentielle pour permettre le développement de la vie à la surface de la terre. Le "trou d'ozone" apparu en 1985 fut la première grande manifestation de l'action de l'homme sur notre planète.

Les précurseurs

2 Les premières observations de l'ozone en France ont concerné la fraction de l'ozone proche du sol, l'ozone troposphérique, grâce aux mesures effectuées dès 1877 à l'observatoire du Parc Montsouris, proche de Paris. Ces données servent aujourd'hui de référence pour démontrer la croissance de ce composant au niveau du sol, sous l'effet de la pollution et notamment de la circulation automobile. L'ozone dans la stratosphère n'a attiré l'attention que plus tard, cette région de l'atmosphère étant longtemps mal connue, car difficile à observer. Cependant vers 1920, Charles Fabry et Henri Buisson, célèbres précurseurs de l'École d'optique en France, ont mesuré l'épaisseur de la couche d'ozone grâce à l'absorption du rayonnement ultraviolet solaire dans l'atmosphère. À leur suite, Jean Cabannes, Jean Dufay, Daniel Challonge, Étienne Vassy en étudient la variabilité et en précisent l'altitude, et, en complément des travaux de Gordon M. B. Dobson en Angleterre, Paul Götz en Suisse et Marcel Nicolet en Belgique, ils mettent sur pied les premiers réseaux d'observation de l'ozone. C'est grâce à ces pionniers que s'est développée en France une discipline, l'aéronomie qui, avec des moyens plus modernes, allait permettre de poursuivre leurs travaux. En effet, à partir des années 1950, l'emploi des fusées sondes et, un peu plus tard des ballons, ouvre la voie à de nouvelles approches pour étudier l'environnement terrestre, en permettant l'observation et la mesure des paramètres de l'atmosphère in situ et au-dessus de l'écran atmosphérique. 1970, année charnière 
3 Le service d'aéronomie du CNRS créé en 1958 par Jean Coulomb, directeur général du CNRS à l'époque, et dont la direction fut très rapidement confié à Jacques Blamont, se proposait d'étudier les processus physico-chimiques et dynamiques gouvernant l'évolution des atomes et molécules formés par l'ionisation et la photodissociation des constituants dans les hautes couches de l'atmosphère sous l'action du rayonnement solaire. Le terme "aéronomie » permettait de distinguer clairement cette région du domaine de la météorologie où la principale source d'énergie provient du rayonnement de la terre et comme on le sait, du piégeage de cette énergie par les gaz à effet de serre. L'ozone stratosphérique se situait parfaitement dans le créneau de recherche de l'aéronomie, mais il fallut quelques années encore pour que la communauté scientifique s'y intéresse. C'est au début des années 1970 qu'apparurent les premières annonces de risques portant sur l'équilibre de l'ozone dans la stratosphère. Tout d'abord en 1970, Paul Crutzen ${ }^{1}$ identifia le rôle potentiel des oxydes d'azote, particulièrement le monoxyde d'azote $\mathrm{NO}$ et le dioxyde $\mathrm{NO}_{2}$ dans la destruction de l'ozone. Or c'est précisément à cette époque que les avions commerciaux supersoniques, volant à très haute altitude, ont été accusés d'émettre ces composés nitrés et donc de mettre en danger la couche d'ozone. Les enjeux économiques et politiques liés au développement du supersonique Concorde ont conduit rapidement à la création de comités d'étude sur les risques encourus : en France c'est le Covos qui, grâce à des financements industriels, soutint ces recherches auxquelles ont participé le Service d'aéronomie (SA) et l'Onera.

Les travaux intensifs sur la couche d'ozone, tant en instrumentation qu'en modélisation ont bénéficié de cette inquiétude pour se développer. Cet intérêt s'est avéré d'autant plus utile que quatre ans plus tard, F. Sherwood Rowland et Mario Molina découvrent le risque supplémentaire posé par les fréons, les fameux chlorofluorocarbones (CFC) utilisés dans les réfrigérateurs et les bombes à aérosols. Ces composés nitrés et chlorés entrent dans des cycles catalytiques de destruction de l'ozone que l'on découvre d'une grande efficacité, notamment dans la haute stratosphère. S'y ajouteront plus tard les composés du brome.

5 Mais le coup fatal est porté par l'annonce en 1985 par des scientifiques anglais et japonais. Leurs observations menées depuis 30 ans à Halley Bay et à Syowa en Antarctique montrent une réduction de moitié de la couche d'ozone dans l'atmosphère polaire pendant le printemps. L'annonce est d'autant plus inattendue que les travaux menés depuis les années 1970 n'avaient pas laissé prévoir une destruction de l'ozone au-dessus de l'Antarctique. Il s'agissait donc d'un phénomène qui n'avait pas été envisagé dans les recherches jusqu'à ce jour. C'est à Susan Solomon² que l'on doit en grande partie l'explication du rôle de la chimie hétérogène autour des nuages stratosphériques polaires dans la destruction de l'ozone antarctique. Les conditions de basse température et de flux UV nécessaires à ces réactions ne sont remplies que dans des conditions présentes en région polaire au printemps.

Les recherches menées par les équipes françaises

Une première mesure de l'ozone en Antarctique avait été faite en 1957 pendant l'Année géophysique internationale par Arlette Vassy, mais n'ayant pas eu de suite, les équipes françaises n'avaient pas les données nécessaires pour détecter l'apparition du trou d'ozone. Cependant, depuis les années 1970, les inquiétudes suscitées par le Concorde avaient entraîné le lancement d'un programme de mesures spectrométriques à bord du Concorde, ainsi que la détection de sa traînée par sondage laser, mais aucune mesure n'eut lieu en région polaire. En revanche, la France a pu, pendant cette période, 
parfaire l'instrumentation et procéder à des campagnes d'inter-comparaison des instruments capables de mesurer l'ozone, aux États-Unis (BIC, 1981-1982) et en France (GLOBUS, 1983 et 1985).

7 La découverte du trou d'ozone au-dessus de l'Antarctique a rapidement suscité la crainte qu'une situation identique ne se produise en Arctique $d u$ fait des populations locales et de nombreuses actions se sont mises en place à l'échelle internationale pour comprendre les mécanismes de destruction de l'ozone en région polaire. Dès 1988, des spectromètres $\mathrm{SAOZ}$ conçus par Jean-Pierre Pommereau (SA) étaient implantés à Dumont d'Urville et à Sodankyla et ils sont actuellement en opération dans 19 stations, dont 10 sous le contrôle du CNRS (resp.: Florence Goutail, SA). Sous l'égide de la commission européenne, des campagnes ont été organisées rapidement pour étudier les processus dans l'Arctique (EASOE en 1991-92, SESAME en 1994, THESEO en 1999...); les équipes françaises y ont participé grâce au concours du CNRS et du CNES avec l'instrumentation au sol et embarqué développé au cours des décennies précédentes: notamment les lidars ou sondage de l'atmosphère par laser, et les spectromètres au sol et embarqués sur ballon, ainsi que les lidars embarqués sur avion et une participation aux mesures par satellite. Les résultats de ces campagnes ont joué un rôle très important dans la compréhension de la destruction de l'ozone arctique et ont imposé l'Europe dans la "course scientifique » face aux États-Unis, signant là une réelle coopération européenne.

8 Parallèlement à l'organisation de ces campagnes ponctuelles et focalisées sur l'Arctique, la nécessité de surveiller sur le long terme l'ozone et les espèces destructrices d'ozone ainsi que les paramètres stratosphériques a entraîné la mise en place d'un réseau international de mesures dans lequel la France a joué un rôle de leader. Ce réseau de surveillance de la stratosphère (NDSC), décidé en 1991 et complété récemment pour la surveillance de la troposphère (NDACC), s'est développé en France dans le cadre de l'IPSL (resp. : Philippe Keckhut). Les stations du réseau comportent des lidars pour la mesure de l'ozone, de la température et des aérosols, des spectromètres UV/visible SAOZ, les uns et les autres développés au SA, les spectromètres IR du Lisa, et des spectromètres micro-ondes de l'observatoire de Bordeaux. La contribution française a permis l'implantation de stations NDACC à différentes latitudes: notamment l'OHP, l'île de la Réunion, Dumont d'Urville, Andoya... La station de l'OHP, première à commencer des mesures à long terme, a même anticipé la reconnaissance à l'échelle internationale du NDSC, puisque les mesures de température par lidar y ont commencé en 1979 et celle de l'ozone par lidar en 1985 (resp. : Marie- Lise Chanin, Alain Hauchecorne, Gérard Mégie et Sophie Godin SA/IPSL).

9 De plus, les instruments embarqués sur ballon, notamment des SAOZ ont permis la mesure fréquente des profils verticaux d' $\mathrm{O}_{3}$, des composés destructeurs d'ozone, $\mathrm{NO}_{2}$, $\mathrm{BrO}$, OClO, IO, et des aérosols en Arctique, aux latitudes moyennes et aux tropiques. Il existe également une version pour des vols de longue durée (de l'ordre du mois) sous MIR (Montgolfière Infrarouge). Très récemment en 2005, une flottille de 27 ballons surpressurisés a volé autour de l'Antarctique pendant des périodes de plusieurs mois sous la direction de François Vial (LMD/IPSL) pour mesurer les paramètres atmosphériques (projet Vorcore).

10 Outre la participation au NDSC et aux campagnes, les laboratoires français ont participé grâce au CNES à la réalisation et à l'exploitation de plusieurs satellites d'étude de la stratosphère à la fois dans le cadre de coopération France-USA avec UARS et dans le 
cadre européen avec Envisat. Le satellite Gomos à bord d'Envisat a été conçu et réalisé et est exploité au SA par Jean-Loup Bertaux et Alain Hauchecorne. Depuis les années 1970, des études théoriques ont été menées au SA, au LMD et au CNRM, sur les mécanismes liés à la destruction de l'ozone par les oxydes d'azote, de brome, de chlore et les nuages stratosphériques polaires, et sur le transport à travers les barrières dynamiques stratosphériques.

11 Elles ont permis d'améliorer la modélisation de la destruction d'ozone grâce aux modèles Reprobus, Mimosa- Chim... et actuellement elles vont permettre l'introduction de la stratosphère dans le modèle couplé du climat, notamment dans LMDz, de façon à intégrer les problèmes posés par les perturbations de l'ozone dans la modélisation du climat.

Vers un avenir serein?

l est quasiment impossible de distinguer, sans entrer dans une présentation très complexe, la contribution spécifique française à la compréhension du problème de l'équilibre de l'ozone et de sa destruction pendant les dernières décennies. Les re cherches ont été menées d'une façon complètement internationale, une très forte contribution de l'Europe pour la recherche dans l'Arctique, et des contributions plus ponctuelles dans l'Antarctique. La combinaison des recherches sur le terrain et en laboratoire ainsi que l'acquisition de base de données, essentielles aujourd'hui pour suivre l'évolution des phénomènes, a été quasi exemplaire, puisque en l'espace d'un peu plus de trois décennies, les problèmes ont été découverts, identifiés, attribués à des causes précises, et mis sous contrôle par une régulation internationale. Il reste encore des points obscurs, notamment sur les échanges entre la troposphère et la stratosphère, et donc sur l'impact du changement climatique sur le comportement de l'ozone et sa récupération.

13 Mais, a priori les conséquences portent surtout sur l'incertitude quant à la date prévue pour le retour à la normale, (vers 2050) mais sauf surprise, ce qui n'est jamais à négliger, la destruction de l'ozone est maintenant comprise et contrôlée.

La participation française aux structures internationales : IOC/IAMAS, WMO-UNEP

14 Les scientifiques français ont toujours été très présents dans les structures internationales relevant de l'ozone stratosphérique. Ce thème est traité dans le cadre de l'Association internationale de météorologie et de sciences de l'atmosphère (IAMAS) par le Comité international de l'ozone (IOC) auquel les scientifiques français ont appartenu depuis son origine en 1929 et dont Gérard Mégie est devenu membre en 1980, vice-président en 1984 et président en 1988 jusqu'à sa disparition en 2004. Sophie Godin Beckmann (SA/IPSL) en est actuellement la vice-présidente. En 1987, le protocole de Montréal qui réglemente les émissions de substances destructrices d'ozone, a été ratifié par la plupart des pays et il a fait l'objet d'amendements successifs pour prendre en compte l'amélioration des connaissances. Pour en contrôler l'efficacité, l'Organisation météorologique mondiale OMM/WMO et le Programme des Nations Unies pour l'environnement PNUE/UNEP ont mis en place une structure chargée à intervalles réguliers de rédiger un rapport sur l'état de la couche d'ozone (l'équivalent du GIEC pour le climat). Ce groupe a publié en 2006 son sixième rapport dans lequel, pour la première fois, on peut annoncer le début de récupération de la couche d'ozone. Gérard Mégie a été l'un des co-présidents de ce groupe pour les rapports publiés en 1998 et 2002, et Marie-Lise Chanin était membre du comité scientifique pour le dernier rapport publié en 2006. De plus, au cours des années, un grand nombre de scientifiques 
français ont contribué à ces rapports, que ce soit en tant qu'auteurs, contributeurs ou rapporteurs de différents chapitres.

\section{NOTES}

1. P. Crutzen, F. S. Rowland et M. Molina ont reçu le prix Nobel de chimie en 1995 pour ces travaux.

2. S. Solomon, médaille nationale de la science des USA en 1999 pour ces travaux.

\section{RÉSUMÉS}

Marie-Lise Chanin décrit les craintes qu'a suscité le fragile équilibre de l'ozone stratosphérique, notamment après la découverte du «trou d'ozone » en Antarctique, en insistant sur le rôle qu'a alors joué la recherche française et en particulier celui du CNRS.

Marie-Lise Chanin describes the fears raised by the very fragile equilibrium of the ozone layer, specially after the discovery of the Antarctic "ozone hole", insisting on the role of French research and on the role of CNRS.

\section{AUTEUR}

\section{MARIE-LISE CHANIN}

Marie-Lise Chanin est directrice de recherche émérite au CNRS. Elle est membre du Service d'aéronomie du CNRS/ Institut Pierre-Simon-Laplace (SA/IPSL), membre correspondant de l'Académie des sciences et membre de l'Académie des technologies. 